\title{
Performances of a Prototype for the ALICE Muon Trigger at LHC
}

\author{
R. Arnaldi, A. Baldit, V. Barret, N. Bastid, G. Blanchard, E. Chiavassa, P. Cortese, P. Crochet, G. Dellacasa, \\ N. De Marco, C. Drancourt, P. Dupieux, A. Ferretti, B. Forestier, C. Insa, F. Jouve, M. Gallio, A. Genoux-Lubain, \\ L. Lamoine, F. Manso, P. Mereu, A. Musso, C. Oppedisano, A. Piccotti, F. Poggio, P. Rosnet, L. Royer, P. Saturnini, \\ E. Scalas, E. Scomparin, F. Sigaudo, G. Travaglia, E. Vercellin, and F. Yermia
}

\begin{abstract}
ALICE, the experiment dedicated to the study of heavy ion collisions at LHC, will be equipped with a forward spectrometer to identify heavy quarkonium states from their decay into muons. The trigger system of the spectrometer consists of four planes of resistive plate chambers, front-end and fast-decision electronics. It is designed to reconstruct muon tracks in a large background environment and to provide a fast trigger signal to the spectrometer. We present results from the test of a trigger prototype that have been carried out with the muon beam of the CERN/SPS at the Gamma Irradiation Facility (GIF). The track finding efficiency as well as the robustness of the system to the uncorrelated background induced on the detector by the GIF source are discussed. Special emphasis is put on timing aspects which are crucial at LHC. A self-consistent method for timing optimization and monitoring is proposed. The findings, although specific to ALICE, are relevant to any muon trigger system operating at LHC.
\end{abstract}

Index Terms-Dimuon, heavy ion collision, resistive plate chamber, timing, trigger.

\section{INTRODUCTION}

$\mathbf{H}$ EAVY quarkonium states provide, via their leptonic decays, one of the most promising observables for the study of the Quark Gluon Plasma (QGP). This has attracted enormous experimental and theoretical activity over the last years (for reviews, see [1], [2]). At LHC, the ALICE detector [3] will measure quarkonia in both dielectron and dimuon channels. Dimuon measurements will be performed by means of a forward spectrometer [4], [5] which is designed to identify the full set of

Manuscript received June 6, 2003; revised October 29, 2003. This work was supported by the "Region Auvergne."

R. Arnaldi, E. Chiavassa, N. De Marco, A. Ferretti, M. Gallio, P. Mereu, A. Musso, C. Oppedisano, A. Piccotti, F. Poggio, E. Scomparin, F. Sigaudo, E. Vercellin, and F. Yermia are with the INFN, Sezione di Torino, Dipartimento di Fisica Sperimentale, Università di Torino, 10125 Torino, Italy.

A. Baldit, V. Barret, N. Bastid, G. Blanchard, P. Crochet, C. Insa, F. Jouve, A. Genoux-Lubain, L. Lamoine, F. Manso, P. Rosnet, L. Royer, and P. Saturnin are with the LPC Clermont-Ferrand, IN2P3/CNRS et Université Blaise Pascal, 63177 Aubière Cedex, France.

P. Cortese, G. Dellacasa, and E. Scalas are with the Dipartimento di Scienze e Tecnologie Avanzate dell' Università del Piemonte Orientale, 15100 Alessandria, Italy.

C. Drancourt is with the Subatech, IN2P3/CNRS, Ecole des mines et Université de Nantes, 44070 Nantes Cedex 03, France.

P. Dupieux and B. Forestier are with the LPC Clermont-Ferrand, IN2P3/CNRS et Université Blaise Pascal, 63177 Aubière Cedex, France (e-mail: dupieux@clermont.in2p3.fr; forestie@clermont.in2p3.fr).

G. Travaglia is with the INFN, Sezione di Torino, 10125 Torino, Italy, and also with the Dipartimento di Fisica dell' Università di Messina, 98166 S. Agata (Messina), Italy.

Digital Object Identifier 10.1109/TNS.2004.828621 quarkonium resonances from the $\phi$ to the $\Upsilon$, with high statistics and high resolution. This spectrometer covers the pseudorapidity range $2.5<\eta<4$. It consists of a front and a small angle absorber, a large dipole magnet, ten high granularity tracking chambers, a muon filter, and a trigger system [4]-[6].

The muon trigger participates to the level 0 of the ALICE general trigger chain. The level 0 is the fastest one, with a total latency of $1.2 \mu \mathrm{s}$. A muon High-Level Trigger (HLT) implemented in computer farms is presently under development but is not described in this note.

The trigger signal is built in three steps. First, single muon tracks with their magnetic deviation are reconstructed by algorithms running in hardware thus allowing to get rid of most of the uncorrelated soft background. Second, a cut on the magnetic deviation (equivalent to the transverse momentum $\mathrm{P}_{\mathrm{T}}$ ) is performed to reject low $\mathrm{P}_{\mathrm{T}}$ muons which originate, to a large extent, from pion and kaon decays. Finally, by combining two opposite-sign muon tracks over the whole acceptance of the setup, resonances such as $\phi, J / \psi$ or $\Upsilon$ are flagged.

Like most of the large area muon triggers recently built or presently being built, the muon trigger of the ALICE detector is based on Resistive Plate Chambers [7] (RPCs). However, in contrast to other LHC experiments [8], [9], it has been decided to operate the RPCs in streamer mode (see [10]-[12] for details about our R\&D). The trigger detector is made of a total of 72 RPCs organized in two stations of two planes of $\sim 30 \mathrm{~m}^{2}$ each. The RPCs are read out on both sides of the gas gap by X-Y orthogonal strips, $10-45 \mathrm{~mm}$ wide and $170-730 \mathrm{~mm}$ long. The total number of channels is 20992 .

In order to improve the timing performances in streamer mode, the dedicated A DUaL Threshold (ADULT) front-end ASIC has been developed [13]-[15]. A time resolution better than $2 \mathrm{~ns}$, with a skew of about $-3 \mathrm{~ns} / \mathrm{kV}$, is obtained in a wide range of operating voltages already starting at the knee of the detector efficiency curve.

The output signals from the front-end, i.e., X-Y bit patterns of the fired strips, are sent to the fast-decision local trigger electronics, which is housed in VME-9U boards. The latency of the local boards is $250 \mathrm{~ns}$. At this level, a complex algorithm [4], $\mathrm{L} 0-\mathrm{X}$ in the bending plane of the dipole and L0-Y in the other direction, is performed in field-programmable gate array (FPGA) circuits, for track finding. Among other criteria, the LO-X and L0-Y algorithms require that a track fires at least three out of the four detector planes in a "road" of a given width. This condition is referenced as the " $3 / 4$ coincidence level" in what fol- 


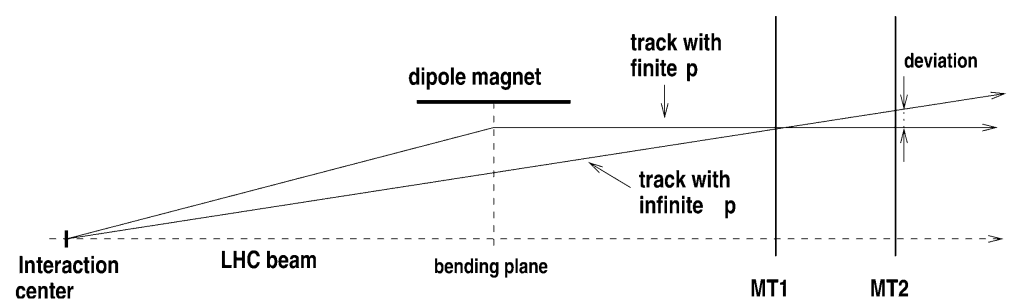

Fig. 1. Principle of a deviation measurement with the ALICE trigger system.

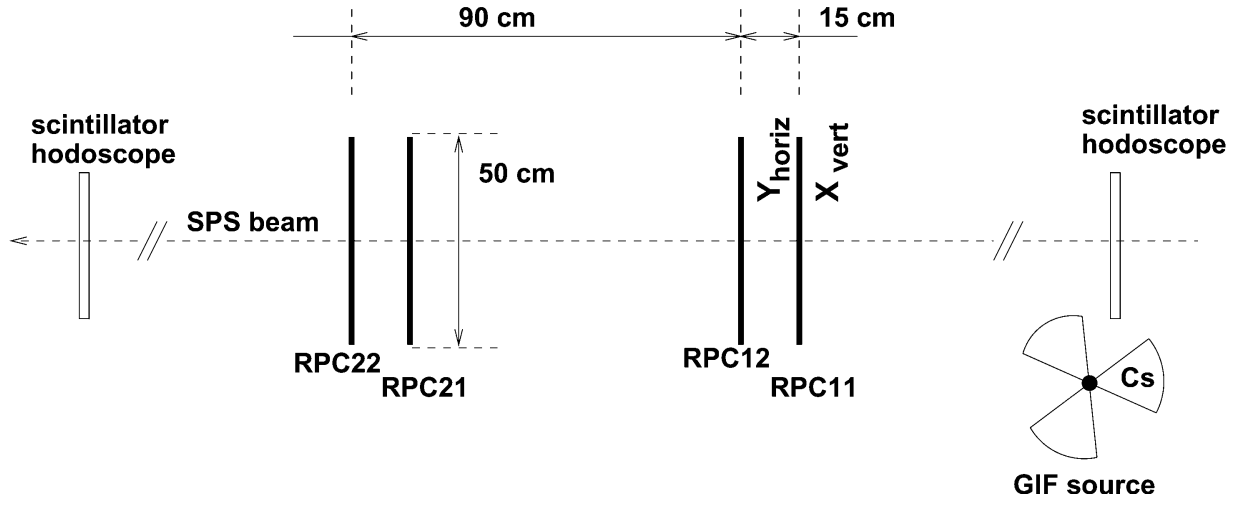

Fig. 2. Sketch of the experimental setup of the minitrigger at GIF.

lows. The $4 / 4$ coincidence level is also implemented and can be activated. The bending in the magnetic field measured between the two trigger stations MT1 and MT2 with respect to the line aiming at the interaction center, as shown in Fig. 1, is also roughly estimated both in sign and amplitude by the LO-X algorithm. Rough cuts on the track deviation, which are preloaded in a lookup table, can be also performed at this level. Each local board receives 128 signals from the front-end electronics, $16 \mathrm{X}-\mathrm{Y}$ signals from each detector plane. The whole trigger system is divided in 234 detection areas, each of them being associated with a local trigger board.

A small area prototype of this setup, called the "minitrigger," has been tested with the CERN/SPS muon beam at the Gamma Irradiation Facility [16] (GIF), during June 2002. In particular, the (muon) track finding efficiency was investigated with and without the uncorrelated background generated by the gamma source of GIF. Of course, no cut on the deviation was performed (by means of the lookup table implemented in the local trigger board) for track-finding efficiency measurements. The fake trigger rate has been measured at various background levels, with beam off, in order to check the robustness of the setup to uncorrelated background. Timing issues have been investigated in detail. A method for timing optimization and monitoring of the muon trigger in ALICE has been deduced from the results of this test.

\section{Description OF THE TEST}

\section{A. Experimental Conditions}

The test has been carried out with a $120 \mathrm{GeV} / \mathrm{c}$ muon beam. The photon flux induced by the intense Cesium source of GIF produces, essentially by Compton effect, uncorrelated background on the four RPCs. The background rate can be varied by means of $\mathrm{Pb}$ absorbers positioned in front of the source. At the setup location, the maximum background rate is 320 (110) $\mathrm{Hz} / \mathrm{cm}^{2}$ on the detector close to (far from) the source. This exceeds by far the expected background level in ALICE [4].

A sketch of the minitrigger setup is shown in Fig. 2. Four $50 \mathrm{~cm} \times 50 \mathrm{~cm}$ RPC planes, geometrically spaced like in ALICE, are positioned in the GIF area perpendicular to the beam direction. Two $30 \mathrm{~cm} \times 30 \mathrm{~cm}$ scintillator hodoscopes are placed upstream and downstream the RPCs, outside the GIF zone, for efficiency measurements.

The RPCs are fluxed in parallel with a gas mixture composed of $51 \% \mathrm{Ar}, 41 \% \mathrm{C}_{2} \mathrm{H}_{2} \mathrm{~F}_{4}, 7 \% \mathrm{i}-\mathrm{C}_{4} \mathrm{H}_{10}$ and $1 \% \mathrm{SF}_{6}$. Each $\mathrm{RPC}$ is read out by $16 \mathrm{X}-\mathrm{Y}$ orthogonal strips. The length and width of the strips are 50 and $2 \mathrm{~cm}$ (pitch size $2.12 \mathrm{~cm}$ ), respectively. The front-end electronics use the ADULT ASIC, the threshold for the streamer detection being $80 \mathrm{mV}$.

The $\mathrm{X}-\mathrm{Y}$ patterns of the fired strips are transmitted to one fast decision local trigger board in LVDS standard along 25-m-long cables. The number of readout channels matches exactly the number of inputs of one local board.

\section{B. Timing Aspects}

The trigger electronics work at a $40-\mathrm{MHz}(25 \mathrm{~ns})$ clock frequency (period). The signals coming asynchronously from the front-end are latched and sampled at this frequency. The trigger algorithm is performed and a new trigger decision is delivered every $25 \mathrm{~ns}$. The signal from the front-end must be as wide as possible but it should not be larger than the clock period in order to belong to a unique and well defined bunch crossing. A width of $20 \mathrm{~ns}$ has been chosen.

From the above considerations, the following is evident.

- Signals from all fired strips of a given event must arrive as synchronously as possible to the trigger electronics in order to avoid efficiency losses, implying strong requirements on the detector and front-end timing quality, 


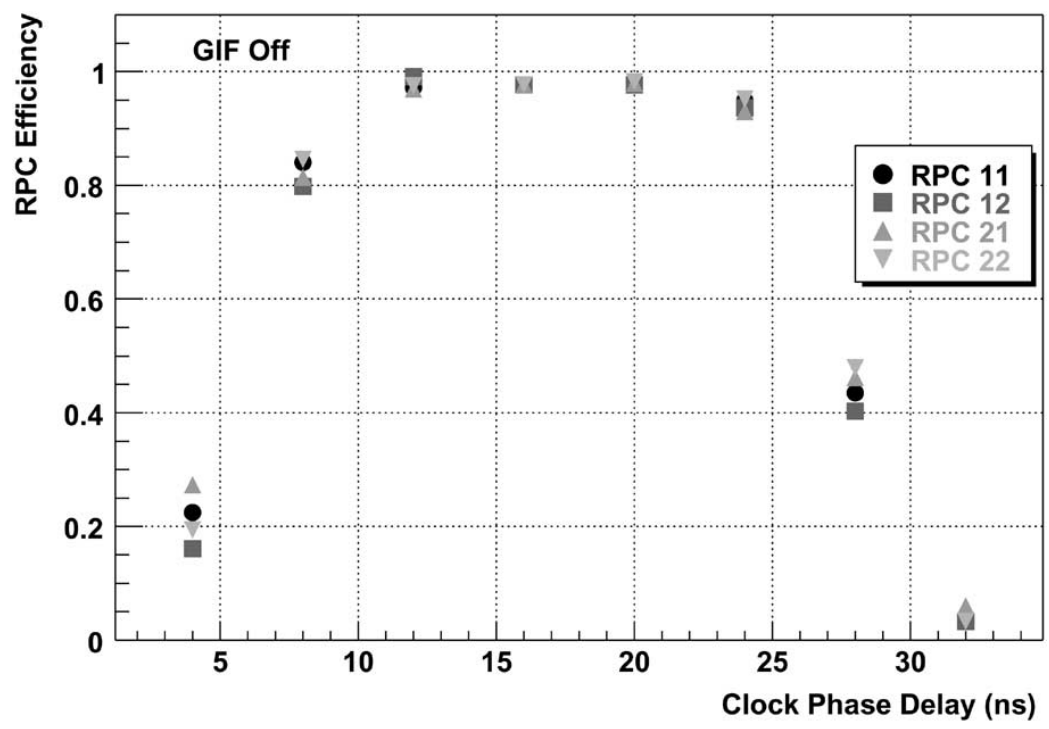

Fig. 3. RPC efficiency at $8 \mathrm{kV}$ (X-planes) with GIF off versus an arbitrary delay applied to the clock phase of the trigger electronics.

cable length from channel to channel, etc. In particular, the distance between the two detector stations must be (and indeed was) compensated for the $3 \mathrm{~ns}$ time of flight difference.

- Interactions must be synchronized with the clock. This will be the case in real working conditions since the LHC clock, which will synchronize the interactions within less than \pm 1 ns [17], will be distributed to the trigger electronics. In this test experiment, the SPS muons and the clock of the electronics were completely asynchronous. To overcome this problem, we selected the muons which are synchronous with the clock within $\pm 2.5 \mathrm{~ns}$ (as explained in the next section). The others are rejected. This is a conservative situation as compared to the LHC one.

- Clock phase must be optimized to latch the signals coming from the front-end with the goal of maximizing the efficiency. This point is abundantly discussed in the following.

\section{Trigger and Data Acquisition (DAQ)}

The DAQ of the minitrigger experiment uses a PC running Labview and connected to a PCI/VME interface. In addition to some standard VME boards like scalers and TDCs used for debugging purposes only, a pipeline memory implemented in the local board is read out. The data stored in this memory include a backup of the current event's bit pattern of the fired strips as well as the trigger decision at different steps in the algorithm.

The data in the DAQ pipeline of the local board are frozen and then read out on occurrence of a trigger signal. Two types of trigger have been used.

- The external trigger delivered by the scintillator hodoscopes when a muon from the beam is detected. This external reference allows to determine and to normalize the efficiency of the minitrigger. For reasons given previously, signals synchronous with the clock (within $\pm 2.5 \mathrm{~ns}$ ) are selected. This is simply done by using narrow signals of about $5 \mathrm{~ns}$. Those in phase with the rising edge of the clock are latched, the others are lost.
- The internal trigger delivered by the local trigger board itself, after the trigger algorithm is performed. This actually corresponds to the trigger as it will be used in ALICE. Its rate at various background levels, with beam off, gives information about the robustness of the system to uncorrelated background.

\section{Performances of THE MinitrigGer}

Results for the RPC efficiency and cluster size, track-finding efficiency, timing, and deviation measurements with the minitrigger are given in the next sections. All raw events have been analyzed without any selection. On all figures, the size of the statistical error bars at $1 \sigma$ are smaller than the symbol size.

\section{A. Timing Optimization}

As aforementioned, muons from the beam are selected in phase with the clock of the trigger electronics. The clock phase must then be tuned and optimized, at the input of the trigger electronics, prior to any measurement. This is illustrated in Fig. 3 which shows the four RPC efficiencies (X-planes), measured at $8 \mathrm{kV}$ operating voltage, as a function of an arbitrary delay applied to the clock phase. The four RPCs reach a $\sim 10$ ns wide plateau at about $98 \%$ efficiency. The same pattern (not shown) is also observed for the Y-planes and any background level. The clock phase is then set at the value corresponding to the middle of the plateau. This is a very robust procedure considering the large safety margin despite all sources of timing dispersion.

\section{B. RPC Efficiency and Cluster Size}

The RPC efficiency is obviously a key feature for the track finding efficiency. It is normalized thanks to the external hodoscope trigger, after optimization of the clock phase as described in the previous section.

RPC efficiency curves measured with GIF off are shown in Fig. 4 for X-planes of the four detectors. Comparable efficiencies (not shown) are achieved for the Y-planes and also with GIF on. The four RPCs exhibit a very homogeneous response with a 


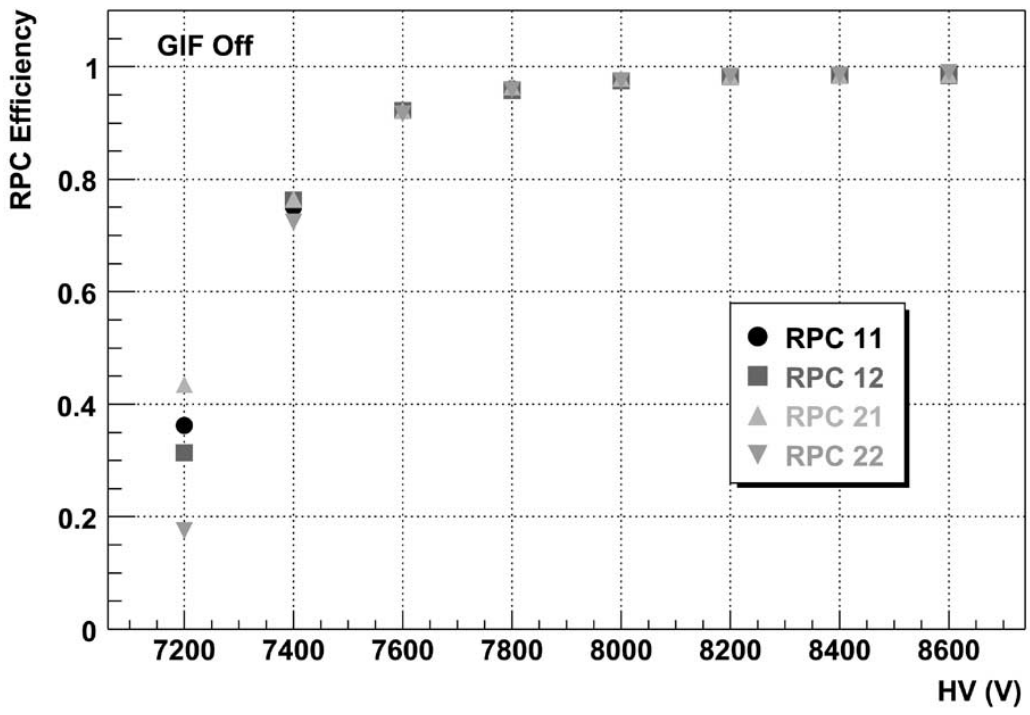

Fig. 4. RPC efficiency (X-planes) versus the operating voltage with GIF off. The normalization of the efficiency is provided by the scintillator hodoscopes.

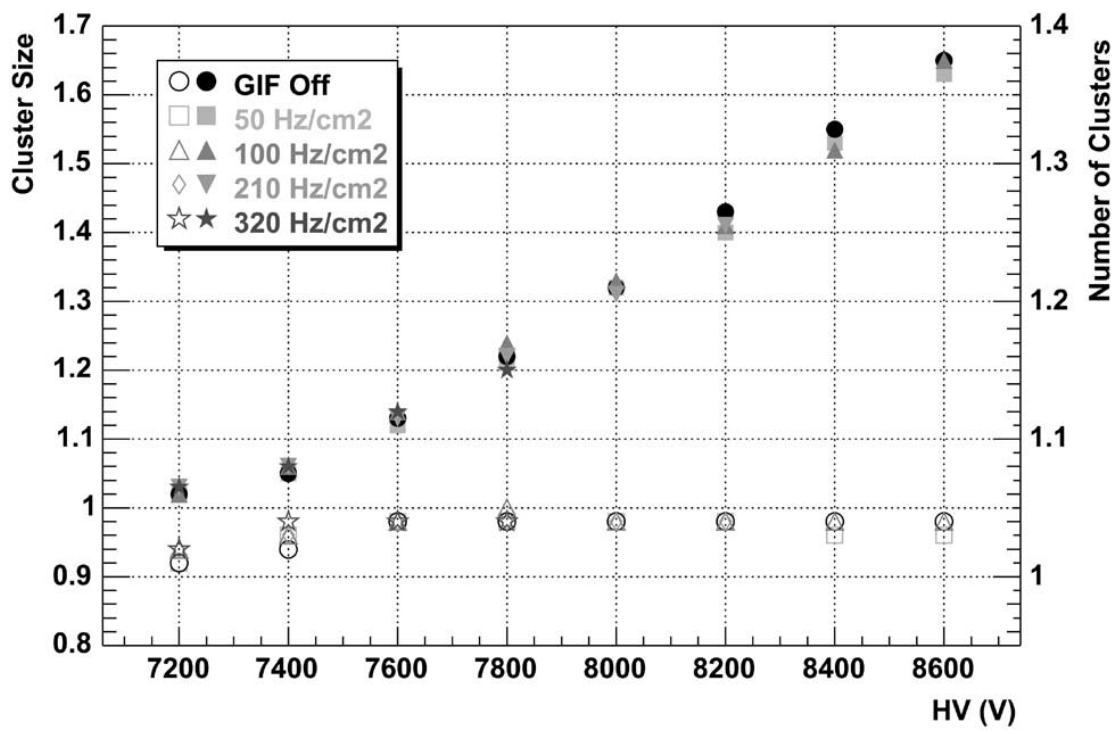

Fig. 5. Cluster size (full symbols, left scale), in strip units, for the X-plane of RPC11 (the closest to the source) as a function of the operating voltage for background rates corresponding to different GIF attenuation settings. Number of reconstructed clusters (open symbols, right scale) in the same conditions.

knee at around $7.6 \mathrm{kV}$ followed by a wide plateau reaching $98 \%$ efficiency.

The cluster size and the number of clusters, for the X-plane of RPC11 (the closest to the source), are presented in Fig. 5 as a function of the operating voltage and for various GIF attenuation settings. The cluster size increases almost linearly with the high voltage, while the number of reconstructed clusters stays constant. No dependence on the background rate is observed. Comparable results were obtained for the other RPCs as well as for the Y-planes.

\section{Track Finding Efficiency Versus RPC Operating Voltage}

The track finding efficiency for various background rates is shown as a function of the RPC operating voltage in Fig. 6 for the $3 / 4$ coincidence level and Fig. 7 for the $4 / 4$ coincidence level. As expected, the track finding efficiency exhibits a shape similar to that of the RPC efficiency. However, the knee and the amplitude of the plateau depend on the coincidence level. Indeed: i) with the $4 / 4$ coincidence level, the knee is moved toward higher voltages and ii) the plateau reaches $98 \%$ and $94 \%$ efficiency with the $3 / 4$ and the $4 / 4$ coincidence level, respectively.

A closer look at the data indicates that there are correlations between the RPC inefficiencies which are attributed to a partial geometrical overlap of the spacers from the different RPC planes, with respect to the beam direction. These correlations explain a large part of the track finding efficiency loss with the $3 / 4$ coincidence level. Indeed, with independent $98 \%$ RPC efficiencies, almost $100 \%$ track finding efficiency would be expected with the $3 / 4$ coincidence level. Such geometrical overlap of the spacers should be avoided to a large extent in ALICE. Note that, on the other hand, the correlation of inefficiencies contributes to increase slightly the track finding efficiency, as 


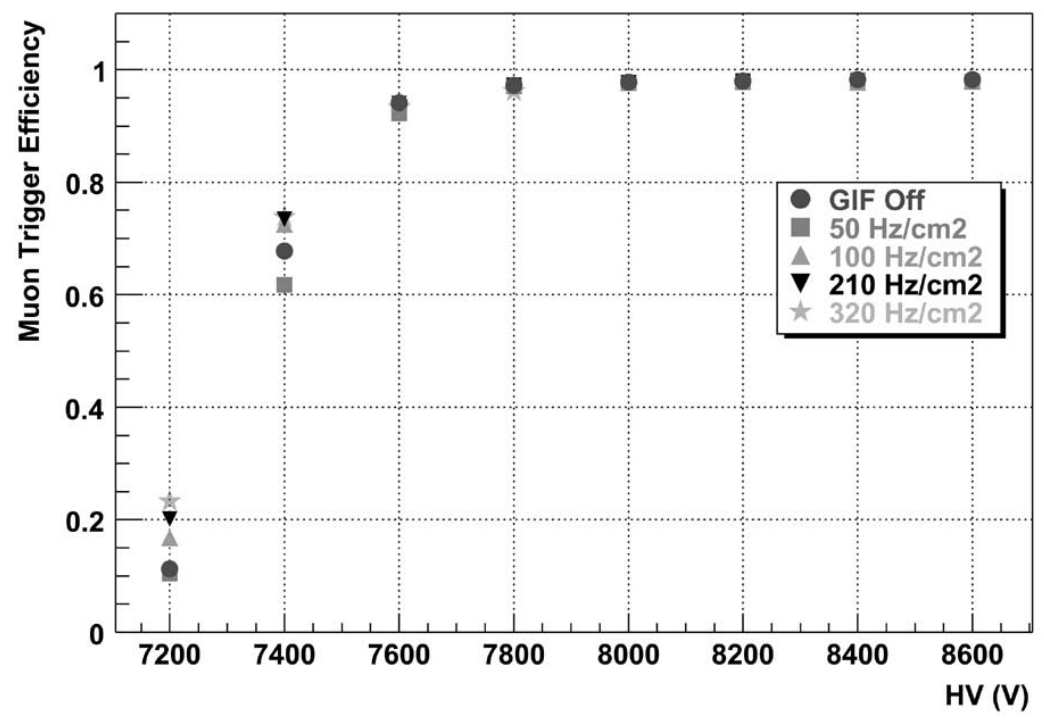

Fig. 6. Track finding efficiency with the $3 / 4$ coincidence level versus the RPC operating voltage for different background rates (indicated values refer to RPC11).

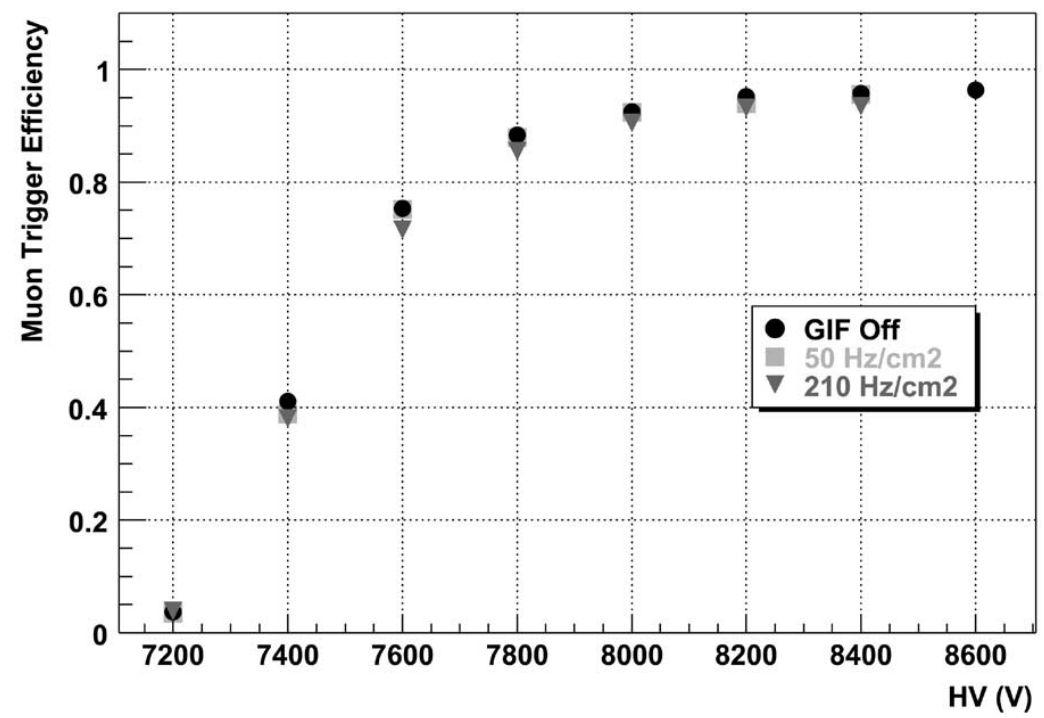

Fig. 7. Track finding efficiency with the $4 / 4$ coincidence level versus the RPC operating voltage for different background rates (indicated values refer to RPC11).

compared again to simple expectations based on independent 98\% RPC efficiencies, with the $4 / 4$ coincidence level.

These experimental results demonstrate clearly that the track finding efficiency is better with the $3 / 4$ than with the $4 / 4$ coincidence level, while the robustness of the system to uncorrelated background is still satisfactory. It can be also seen from Fig. 6 that, for large background rates, there is no indication for an increase of the track finding efficiency which could have been caused by fake track finding. Note that the dispersions observed in Fig. 6 at the lowest voltages for different background rates can be attributed to variations of ambient temperature and atmospheric pressure during data taking.

\section{Track Finding Efficiency Versus Clock Phase}

The track finding efficiency with the $3 / 4$ coincidence level is shown in Fig. 8 as a function of the clock phase. Results obtained for various operating voltages and with GIF on and off are presented.
Here again, since the track finding efficiency and the RPC efficiency are closely correlated, the same kind of efficiency curves are obtained as a function of the clock phase. By comparing the upper and lower plots in Fig. 8, it is evident that the optimization of the clock phase does not depend on the background rate. Note that a slight shift of the plateau toward smaller delay values can be seen for increasing voltages. This can be attributed to a faster detector response at higher voltages which was found to be $-3 \mathrm{~ns} / \mathrm{kV}$ in these running conditions in earlier measurements [15].

\section{E. Deviation Measurements}

The track deviation measured by the minitrigger is close to zero (Fig. 9) since the setup is orthogonal to the beam direction. It is expressed in bit unit, where one bit corresponds roughly to the half width of a strip, thanks to the declustering algorithm performed in the local board [4]. Note that the alignment accuracy between strips of the four detector planes was not better than a 

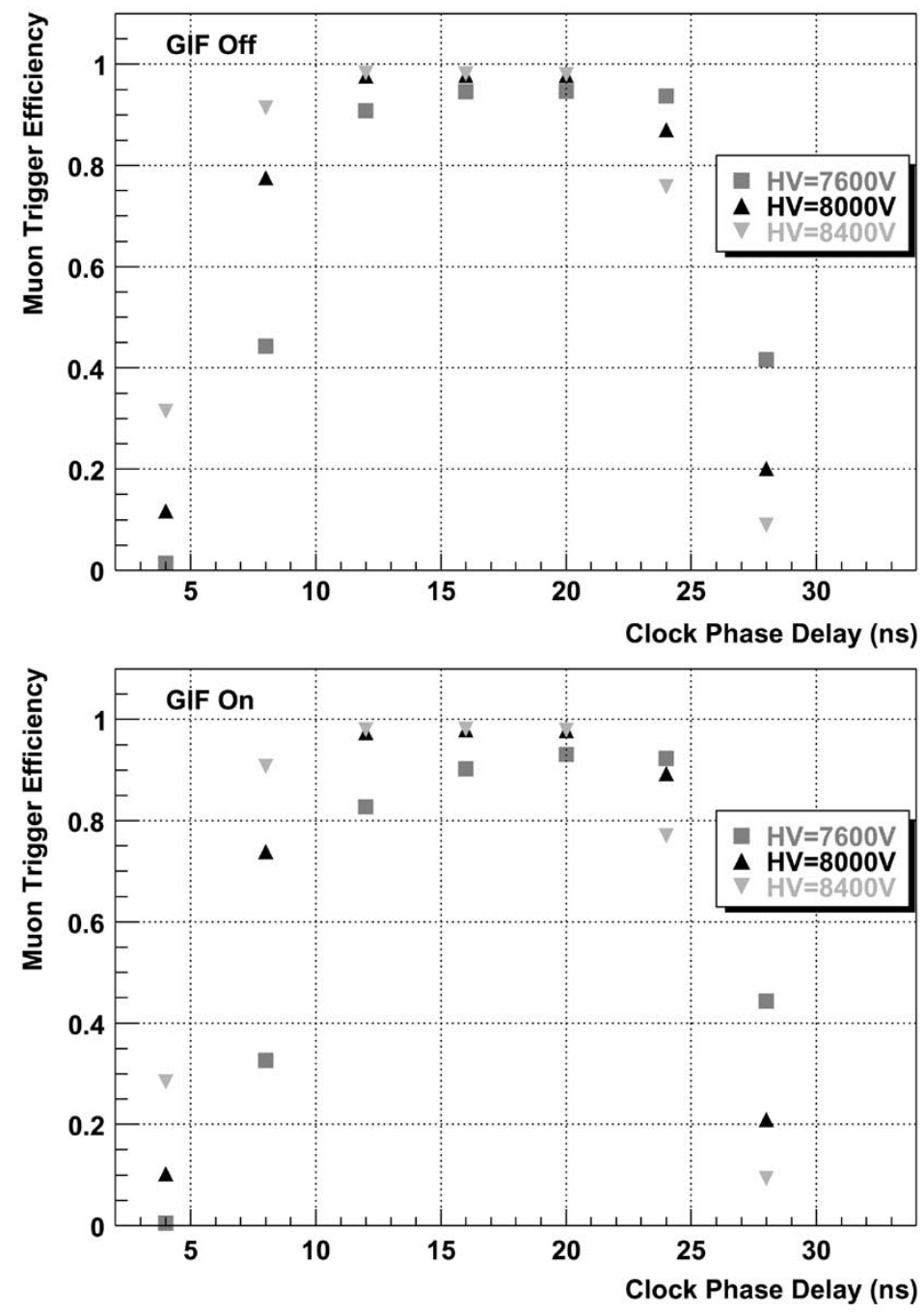

Fig. 8. Track finding efficiency with the $3 / 4$ coincidence level versus the clock phase delay. The data are shown for three operating voltages with GIF off (top) and GIF on (bottom, corresponding to $210 \mathrm{~Hz} / \mathrm{cm}^{2}$ on RPC11).

few millimeters in this test, which may explain the asymmetry of the track deviation spectrum. The accuracy of the measured deviation can be roughly translated, in ALICE conditions, into a transverse momentum $\mathrm{P}_{\mathrm{T}}$ resolution of $10 \%$, at $\mathrm{P}_{\mathrm{T}}=1 \mathrm{GeV} / \mathrm{c}$ (typical cut).

\section{F. Timing Optimization and Monitoring in ALICE}

The determination of the optimum clock phase and its monitoring will be of crucial importance in ALICE. Within this test experiment, such an optimization can be easily determined thanks to the external reference provided by the scintillator hodoscopes.

In fact, the optimization of the clock phase without any external reference can be deduced from the self-consistently calculated ratio of the trigger yields with the $4 / 4$ over the $3 / 4$ coincidence level, as shown in Fig. 10. It is centered at the optimal value of the clock phase delay with no apparent dependence on the background rate.

The redundancy provided by the four detector planes for track finding is directly exploited while calculating this ratio. It has been also checked that this ratio is not sensitive to fluctuations
TABLE I

FAKE TRIGgER RATE (UPPER LIMITS) FOR TwO GIF AtTenuation Settings, With Beam OfF

\begin{tabular}{l|c|c}
\hline & $\begin{array}{c}\text { background rate } \\
\left(\mathrm{Hz} / \mathrm{cm}^{2}\right) \\
\text { on RPC11 (close) }\end{array}$ & $\begin{array}{c}\text { fake trigger rate } \\
\text { per minute }\end{array}$ \\
\hline GIF (with attenuation) & 50 & $<0.3$ \\
GIF (no attenuation) & 320 & $<3$ \\
\hline
\end{tabular}

of the beam intensity because both numerator and denominator vary in the same way. In addition, its absolute value gives indications on the track finding efficiency itself.

In ALICE, this piece of information will be available locally since this ratio can be calculated for each of the 234 local trigger boards. This looks very promising and could help for the timing optimization process. It still needs to be further investigated.

\section{G. Robustness to Uncorrelated Background}

The fake trigger rates obtained in internal trigger mode, with beam off, are shown in Table I for two GIF attenuation settings. The operating voltage is $8 \mathrm{kV}$ and the $3 / 4$ coincidence level is required. No deviation cut is applied. Only upper limits are 


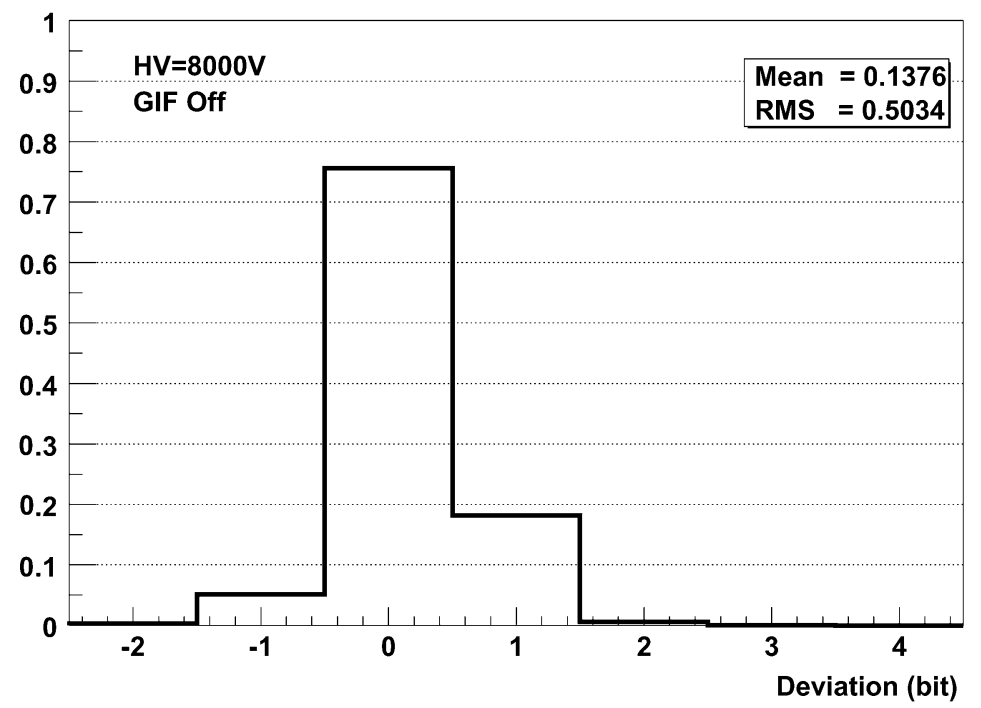

Fig. 9. Track deviation, in bit unit, for a RPC operating voltage of $8 \mathrm{kV}$ with GIF off. The fraction of events with a given deviation is indicated by the vertical scale.
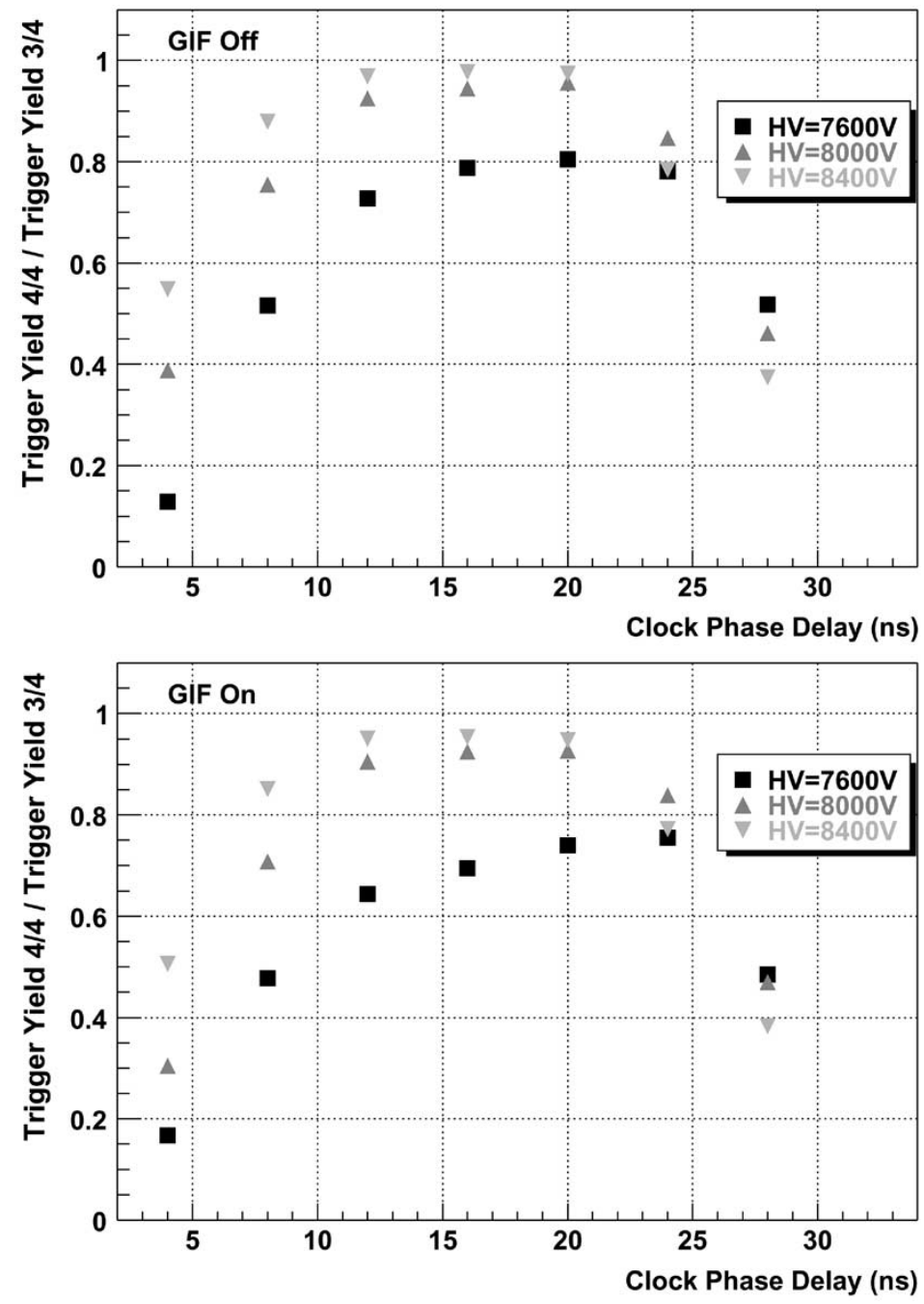

Fig. 10. Ratio of the trigger yields with the $4 / 4$ over the $3 / 4$ coincidence level versus the clock phase delay. It is shown for three operating voltages with GIF off (top) and GIF on (bottom, corresponding to $210 \mathrm{~Hz} / \mathrm{cm}^{2}$ on RPC11). 
given because the statistics is rather poor, of the order of a few tens of events.

Less than three events per minute were recorded at the maximum achievable background rate $\left(320 \mathrm{~Hz} / \mathrm{cm}^{2}\right.$ on RPC11). This is in agreement with simple estimates of the expected event count created by uncorrelated background such as the one at the GIF.

In reduced background conditions $\left(50 \mathrm{~Hz} / \mathrm{cm}^{2}\right.$ on $\mathrm{RPC} 11$, comparable to the maximum estimated yields from simulation in ALICE), the measured event rate is one order of magnitude above the expected one from pure combinatorial background. Indeed, it has been checked that most events are induced by "horizontal" cosmic rays.

These findings clearly demonstrate the robustness of the setup to uncorrelated background. The extrapolation of this result to ALICE conditions is however not straightforward because, at the LHC, the background is expected to be more complex and time-correlated with the signal. There are other more relevant background sources contributing to the trigger rates in ALICE, which are essentially muon tracks from pions and kaons decay. As discussed in detail in [4], soft background on top of these various sources contributes to increase slightly the fake trigger rate in $\mathrm{ALICE}$.

\section{CONCLUSION}

The ALICE muon spectrometer will need to be equipped with a high-performance trigger to select quarkonium states in heavy ion collisions at LHC. The performances of a small scale prototype including most of the final equipments developed for the ALICE muon trigger have been investigated in detail. It is found that the RPC detector efficiency as well as the track finding efficiency reach a $98 \%$ plateau, regardless of the background rate, up to $320 \mathrm{~Hz} / \mathrm{cm}^{2}$. The timing quality of the detector associated with its ADULT front-end electronics is responsible for this excellent result. A deep investigation of the timing aspects has also demonstrated that, despite all sources of timing dispersion, a good safety margin is left on the timing optimization. It is shown that this optimization can be performed self-consistently, without any external reference, from the calculation of the ratio of the trigger yields with the $4 / 4$ over the $3 / 4$ coincidence level. This simple and powerful method should help the time tuning process in ALICE. Finally, the robustness of the setup against a high uncorrelated background has been established. All these findings, which can also be relevant to the other LHC experiments, are an encouraging step toward the construction phase of the ALICE muon trigger.

\section{ACKNOWLEDGMENT}

The authors would like to thank G. Alfarone, M. L. Mercier, C. Fayard, P. Ottria, B. Ronfet, E. Sahuc, and G. Savinel for the technical help they provided.

\section{REFERENCES}

[1] H. Satz, "Color deconfinement in nuclear collisions," Rep. Prog. Phys., vol. 63, p. 1511, 2000.

[2] R. Vogt, "J/PSI production and suppression," Phys. Rep., vol. 310, pp. 197-260, 1999.

[3] Tech. Proposal, Dec. 1995. "Addendum to the Technical Proposal" CERN/LHCC 96-32, Oct., 1996.

[4] “Technical Design Report," ALICE Collaboration, CERN/LHCC 99-22, Aug. 1999.

[5] "Addendum to the Technical Design Report," ALICE Collaboration, CERN/LHCC 2000-046, Dec. 2000

[6] R. Arnaldi et al., "The ALICE dimuon trigger: Overview and electronics prototypes," Nucl. Instrum. Methods, vol. A456, pp. 126-131, 2000.

[7] R. Santonico and R. Cardarelli, "Development of resistive plate counters," Nucl. Instrum. Methods, vol. 187, pp. 377-380, 1981.

[8] "Technical Design Report," ATLAS Collaboration, CERN/LHCC 97-22, May 1997.

[9] “Technical Design Report," CMS Collaboration, CERN/LHCC 97-32, Dec. 1997.

[10] R. Arnaldi et al., "A low-resistivity RPC for the ALICE dimuon arm," Nucl. Instrum. Methods, vol. A451, pp. 462-473, 2000.

[11] R. Arnaldi et al., "Ageing tests on the low-resistivity RPC for the ALICE dimuon arm," Nucl. Instrum. Methods, vol. A508, pp. 106-109, 2003.

[12] R. Arnaldi et al., "Spatial resolution of RPC in streamer mode," Nucl. Instrum. Methods, vol. A490, pp. 51-57, 2002.

[13] R. Arnaldi et al., "A dual threshold technique to improve the time resolution of RPC in streamer mode," Nucl. Instrum. Methods, vol. A457, pp. 117-125, 2001

[14] L. Royer, G. Bohner, and J. Lecoq, "A front-end ASIC for the dimuon arm trigger of the ALICE experiment," in Proc. 6th Workshop Electronics for LHC Experiments, Cracow, Poland, Sept. 2000, pp. 323-327. ALICE Collaboration.

[15] P. Dupieux, "A new front-end for better performances of RPC in streamer mode," Nucl. Instrum. Methods, vol. A508, pp. 185-188, 2003. ALICE Collaboration.

[16] S. Agosteo et al., "A facility for the test of large area muon chambers at high rates," Nucl. Instrum. Methods, vol. A452, pp. 94-104, 2000.

[17] “CERN/PS 2001-011 (DR),”, Apr. 2001. 